\title{
Low-dose dobutamine stress myocardial contrast echocardiography for the evaluation of myocardial microcirculation and prediction of overall cardiac function recovery
}

\author{
YUNJIA LIN $^{1}$, XIN GUAN $^{1}$, KAI REN $^{2}$, YANBO ZHU $^{1}$, YAPING LU $^{1}$ and YANWEN SHANG ${ }^{1}$ \\ ${ }^{1}$ Department of Ultrasound; ${ }^{2}$ Cardiac Intensive Care Unit, Tianjin Chest Hospital, Tianjin 300222, P.R. China
}

Received August 12, 2019; Accepted January 27, 2020

DOI: $10.3892 /$ etm.2020.8813

\begin{abstract}
The study aimed to investigate the role of low-dose dobutamine stress myocardial contrast echocardiography (MCE) in evaluating myocardial local microcirculation and predicting cardiac function recovery in patients with myocardial infarction. A total of 50 patients with acute myocardial infarction (AMI) were enrolled in the present study. Positron emission tomography was used as a gold standard to determine viable/non-viable myocardial segments in infarcted myocardial region. MCE and dobutamine stress MCE were carried out $72 \mathrm{~h}$ after PCI. MCE was carried out again to evaluate myocardial condition at 6 months after PCI. As compared with normal myocardial segments, resting MCE revealed a significant decrease of the values of $\mathrm{A}$ (the peak intensity of the time-perfusion intensity curve, reflecting the myocardial blood volume), $\beta$ (the slope of the curve, reflecting the myocardial blood flow (MBF) velocity) and $\mathrm{A} x \beta$ (reflecting $\mathrm{MBF}$ ) of viable and non-viable myocardial segments. After being challenged by dobutamine, the values of $A, \beta$ and $A x \beta$ of normal coronary blood supply areas were significantly increased; while the segments A and A x $\beta$ of viable myocardium were markedly decreased. Patients were further divided into two groups based on the changes in the contrast-enhanced index (CSI) following dobutamine loading. In the dobutamine stress echocardiography-positive group (the CSI increased or decreased by $>0.2$ ), the left ventricular ejection fraction was significantly increased and pro-B-type natriuretic peptide significantly decreased at 6 months following intervention. Low-dose dobutamine stress MCE was indicated to be an
\end{abstract}

Correspondence to: Dr Xin Guan, Department of Ultrasound, Tianjin Chest Hospital, 261 Taierzhuang South Road, Jinnan, Tianjin 300222, P.R. China

E-mail: guanxin2458@126.com

Key words: myocardial local microcirculation, left ventricular function, myocardial contrast echocardiography, dobutamine stress echocardiography, percutaneous coronary intervention, acute myocardial infarction effective method to evaluate myocardial microcirculation perfusion in patients with AMI following PCI. In addition, CSI, as a simple semi-quantitative index, may predict left ventricular function in patients with AMI.

\section{Introduction}

Percutaneous coronary intervention (PCI) is one of the most effective methods for the treatment of acute myocardial infarction (AMI) (1). Cardiac catheterization may be used to clear the narrowed or even occluded coronary lumen, thereby improving myocardial perfusion. However, certain studies have indicated that in the case of impaired coronary microcirculation, even if cardiac revascularization is successful, part of the blood flow to myocardial tissue may not fully return to normal, which may lead to poor recovery of left ventricular function (2).

Myocardial contrast echocardiography (MCE) is a technique using microbubble distribution within the intravascular space to reveal the status of microvascular perfusion (3). It enables direct and visual assessment of microvascular perfusion. MCE has been used to predict the prognosis for patients with myocardial infarction $(4,5)$. MCE is more objective to evaluate myocardial ischemia as compared with normal echocardiography that visually observes the presence or absence of segmental motor abnormalities. Dobutamine acts on the $\beta 1$ and $\beta 2$ receptors and has a different effect on the damaged microcoronary and normal microcoronary vessels. Dobutamine is able to activate viable myocardium and may induce more severe myocardial ischemia. Through observing changes in the intramyocardial development of the ultrasound enhancer under dobutamine stress, surviving myocardium may be identified more objectively and sensitively. Dobutamine is increasingly being used during MCE, named as dobutamine stress MCE, and may significantly improve the detection rate of myocardial ischemia and injury in patients with coronary heart disease (CHD) $(6,7)$. However, there is currently a lack of research into the application of stress MCE following PCI, its role in myocardial microcirculation evaluation and the prediction of subsequent cardiac function recovery. In the present study, the microcirculation of 50 patients with AMI who underwent PCI using MCE and low-dose stress MCE was 
assessed and the cardiac function at 6 months following PCI using MCE was also evaluated.

\section{Materials and methods}

Patients. From June 2016 to August 2017, 50 patients (29 males and 21 females) with AMI who underwent PCI were enrolled in the present study. The characteristics of the patients are listed in Table I. An appropriate stent length and diameter were selected for all patients. The age of the patients ranged from 45 to 76 years, with an average age of $61.3 \pm 12.2$ years. The inclusion criteria were as follows: i) Persistent chest pain and elevated troponin I; ii) electrocardiogram with new myocardial ischemia changes, i.e. new ST segment changes; iii) segmental wall motion abnormalities from echocardiography; and iv) PCI performed within $24 \mathrm{~h}$ of the occurrence of chest pain. Patients were excluded if they met the following exclusion criteria: i) A congenital heart disease or acute heart failure; ii) allergy to contrast agent; iii) malignant arrhythmia and severe atrioventricular block; and iv) slow blood flow or no reflow following PCI. The characteristics of all of the patients are provided in Table I.

Positron emission tomography (PET). PET was used as the gold standard for viable myocardium detection. PET was performed within 3 days after stress MCE. According to the PET results, the myocardial segments were divided into the following groups: Normal myocardium group (segments with normal perfusion and metabolism), viable myocardium group (segments with a decrease in perfusion but with a normal metabolism) and non-viable myocardium group (segments with a defected perfusion and metabolism).

MCE. MCE was performed within $72 \mathrm{~h}$ following PCI. A two-dimensional echocardiogram was performed prior to $\mathrm{MCE}$ and SonoVue (Bracco Imaging S.p.A) was used as the contrast agent. A total of $59 \mathrm{mg}$ SonoVue was diluted in $20 \mathrm{ml}$ normal saline and shaken for $20 \mathrm{sec}$ to obtain a white microbubble suspension. Subsequently, the diluted SonoVue contrast agent was injected into the upper limb anterior wall vein at a constant speed of $1.5 \mathrm{ml} / \mathrm{min}$. After the contrast agent was stabilized in the myocardial image for 2-3 min, high-mechanical index ultrasound beam emission was used to destroy the contrast microbubbles in the myocardium, and subsequently, the reperfusion process of the microbubbles was observed in the apical view of the heart with a low mechanical index $(<0.2)$. Patients were prevented from using $\beta$-blockers and drugs that may affect myocardial contractility $24 \mathrm{~h}$ prior to MCE.

Low-dose dobutamine stress MCE. Low-dose dobutamine was injected at an initial dose of $5 \mu \mathrm{g} / \mathrm{kg} / \mathrm{min}$ and subsequently increased to $10 \mu \mathrm{g} / \mathrm{kg} / \mathrm{min}$ and then $20 \mu \mathrm{g} / \mathrm{kg} / \mathrm{min}$ every three minutes. Any changes in heart rate and blood pressure were observed and MCE was performed a second time using the same settings after reaching the loading dose.

Data analysis/image interpretation. Semi-quantitative analysis of MCE was performed using the following criteria: i) Uniform and sufficient contrast agent display and good perfusion (1 point); ii) sparse contrast agent display and
Table I. Clinical characteristics of patients $(n=50)$.

\begin{tabular}{lc}
\hline Item & Value \\
\hline Age (years) & $61.3 \pm 12.2$ \\
Male sex & $29(58)$ \\
History of smoking & $22(44)$ \\
Hyperlipidemia & $26(52)$ \\
Diabetes & $19(38)$ \\
Hypertension & $28(56)$ \\
Artery affected by infarct & \\
Front descending branch & $38(76)$ \\
Rotating branch & $4(8)$ \\
Right crown & $8(16)$ \\
\hline
\end{tabular}

Values are expressed as the mean \pm standard deviation or $\mathrm{n}(\%)$.

weak perfusion or partial and flaky perfusion ( 0.5 point $)$; and iii) contrast agent filling defect or no perfusion ( 0 point). The semi-quantitative index was statistically analyzed using the contrast-enhanced index (CSI), which is calculated by dividing the sum of the relevant segmental angiographic scores by the number of segments.

Quantitative analysis of MCE was also performed. The change in echo intensity $(\mathrm{dB})$ of the contrast agent microbubble signal in myocardial tissue over time was analyzed by placing the region of interest (ROI) in the center of the wall of each segment (the ROI size was set to a standard of $5 \mathrm{~mm}^{2}$ ). The endometrium, epicardium and papillary muscles were avoided when the area to be analyzed was selected. The QLAB quantification software (Philips, version 10.5) automatically generates the time-perfusion intensity curve and fits the function $Y=A \times\left(1-e^{-\beta t}\right)+C$, where $A$ is the peak intensity of the curve, reflecting the myocardial blood volume, $\beta$, is the slope of the curve, reflecting the myocardial blood flow (MBF) velocity and $\mathrm{A} x \boldsymbol{\mathrm { x }} \beta$ reflects the $\mathrm{MBF}(8,9)$.

Statistical analysis. Data were analyzed using SPSS v22.0 statistical software (IBM, Corp.). The distribution of data was analyzed by Shapiro-Wilk test, and normally distributed data were expressed as the mean \pm standard deviation. Categorical variables are expressed as n (\%). Differences between two independent groups were determined using Student's t-test, while those among three groups were assessed using one-way analysis of variance followed by a Newman-Keuls post hoc test. $\mathrm{P}<0.05$ was considered to indicate a statistically significant difference.

\section{Results}

Myocardial segment collection and adverse effects. A total of 475 myocardial segments were collected from the normal coronary blood supply area, used as normal myocardium control. In the infarcted coronary blood supply area, 264 myocardial segments were collected, including 171 viable myocardial segments and 93 non-viable myocardial segments. No adverse effects, including abnormal heart rate, abnormal 
Table II. Comparison of microcirculation between normal myocardium, viable myocardium and non-viable myocardium at $72 \mathrm{~h}$ after surgery.

\begin{tabular}{|c|c|c|c|c|c|}
\hline Item & Normal myocardium $(n=475)$ & Viable myocardium $(n=171)$ & Non-viable myocardium $(n=93)$ & F-value & P-value \\
\hline$A(d b)$ & $8.47 \pm 2.03$ & $6.86 \pm 1.82^{\mathrm{a}}$ & $1.87 \pm 0.72^{\mathrm{a}}$ & 160.215 & $<0.001$ \\
\hline$\beta(1 / \mathrm{sec})$ & $1.21 \pm 0.43$ & $0.95 \pm 0.33^{\mathrm{a}}$ & $0.43 \pm 0.22^{\mathrm{a}}$ & 118.573 & $<0.001$ \\
\hline$A \times \beta(d b / s e c)$ & $10.15 \pm 3.35$ & $8.68 \pm 2.56^{\mathrm{a}}$ & $1.16 \pm 0.64^{\mathrm{a}}$ & 125.261 & $<0.001$ \\
\hline
\end{tabular}

Values are expressed as the mean \pm standard deviation. ${ }^{a} \mathrm{P}<0.05$ vs. normal myocardium group. MBF, myocardial blood flow. A, peak intensity of the time-perfusion intensity curve, reflecting the myocardial blood volume; $\beta$, slope of the time-perfusion intensity curve, reflecting the MBF velocity; $\mathrm{A} x \beta$, measure of MBF.

Table III. Comparison of microcirculation prior to and after dobutamine loading.

\begin{tabular}{|c|c|c|c|c|c|c|}
\hline \multirow[b]{2}{*}{ Item } & \multicolumn{2}{|c|}{ Normal myocardium $(n=475)$} & \multicolumn{2}{|c|}{ Viable myocardium $(n=171)$} & \multicolumn{2}{|c|}{ Non-viable myocardium $(\mathrm{n}=93)$} \\
\hline & Prior to loading & After loading & Prior to loading & After loading & Prior to loading & After loading \\
\hline$A(d b)$ & $8.47 \pm 2.03$ & $15.42 \pm 2.99^{\mathrm{a}}$ & $6.86 \pm 1.82$ & $4.39 \pm 1.15^{\mathrm{a}}$ & $1.87 \pm 0.72$ & $1.82 \pm 0.68$ \\
\hline$\beta(1 / \mathrm{sec})$ & $1.21 \pm 0.43$ & $2.27 \pm 0.57^{\mathrm{a}}$ & $0.95 \pm 0.33$ & $1.07 \pm 0.51$ & $0.43 \pm 0.22$ & $0.57 \pm 0.27$ \\
\hline $\mathrm{A} \times \beta(\mathrm{db} / \mathrm{sec})$ & $10.15 \pm 3.35$ & $35.67 \pm 5.47^{\mathrm{a}}$ & $8.68 \pm 2.56$ & $7.33 \pm 2.04^{\mathrm{a}}$ & $1.16 \pm 0.64$ & $1.03 \pm 0.26$ \\
\hline
\end{tabular}

${ }^{a} \mathrm{P}<0.05$ vs. prior to loading. Values are expressed as the mean \pm standard deviation. MBF, myocardial blood flow. A, peak intensity of the time-perfusion intensity curve, reflecting the myocardial blood volume; $\beta$, slope of the time-perfusion intensity curve, reflecting the MBF velocity; $\mathrm{A} x \beta$, measure of $\mathrm{MBF}$.
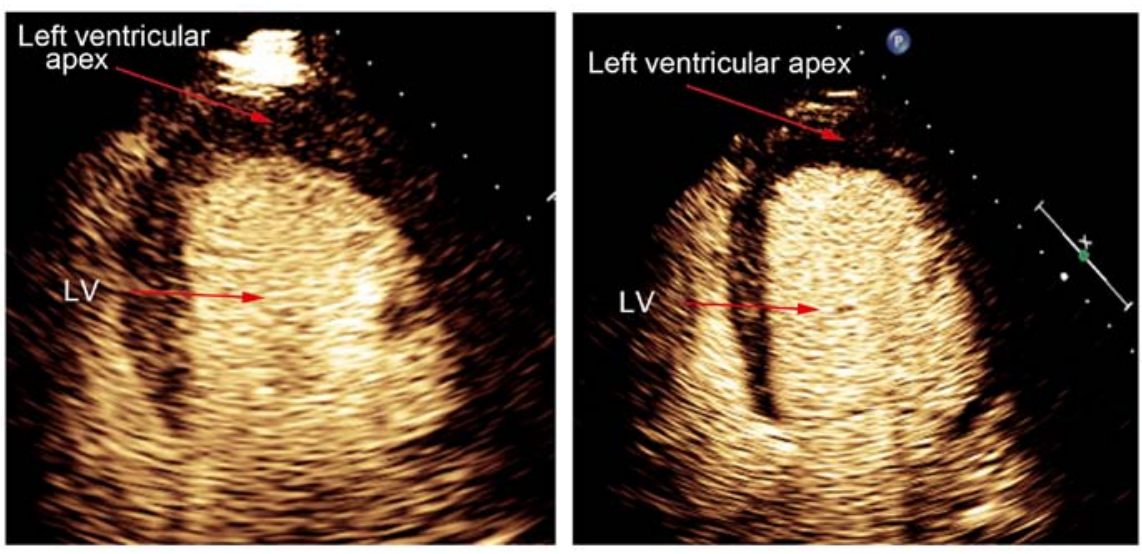

Figure 1. Increased contrast agent filling defect after dobutamine stress in viable myocardial segments. Left panel, viable myocardium prior to dobutamine loading; right panel, viable myocardium after dobutamine loading. LV, left ventricle.

blood pressure, chest tightness, belching or dizziness, were observed during MCE.

Evaluation of microcirculation at $72 \mathrm{~h}$ after PCI. Quantitative analysis revealed that $A, \beta$ and $A \times \beta$ of the normal coronary blood supply area were significantly larger compared with those in the viable myocardium segment and the non-viable myocardium segment (Table II).

The microcirculation was also investigated following low-dose dobutamine loading. Quantitative analysis revealed that dobutamine loading significantly increased the values of $\mathrm{A}, \beta$ and $\mathrm{A} \times \mathrm{x}$ of the normal coronary blood supply area.
However, in the viable myocardium, the segments $\mathrm{A}$ and $\mathrm{A} x \boldsymbol{\mathrm { x }}$ were markedly decreased after dobutamine loading (Table III); there was no significant change for segment $\beta$. As shown in Fig. 1, contrast agent filling defect was enhanced after stress test in viable myocardial segment. Furthermore, no significant changes were observed in the values of $A, \beta$ and $A x \beta$ of the non-viable myocardium after loading (Table III).

Recovery of left ventricular function 6 months after PCI. A total of 6 months following PCI, the values of A, $\beta$ and A $x \beta$ of the viable myocardium group were significantly increased compared with those at $72 \mathrm{~h}$ following surgery; while no 
Table IV. Comparison of microcirculation between 6 months and $72 \mathrm{~h}$ after surgery.

\begin{tabular}{|c|c|c|c|c|c|c|}
\hline \multirow[b]{2}{*}{ Item } & \multicolumn{2}{|c|}{$\begin{array}{c}\text { Normal } \\
\text { myocardium }(n=475)\end{array}$} & \multicolumn{2}{|c|}{$\begin{array}{c}\text { Viable } \\
\text { myocardium }(n=171)\end{array}$} & \multicolumn{2}{|c|}{$\begin{array}{c}\text { Non-viable } \\
\text { myocardium }(n=93)\end{array}$} \\
\hline & $72 \mathrm{~h}$ & 6 months & $72 \mathrm{~h}$ & 6 months & $72 \mathrm{~h}$ & 6 months \\
\hline$A(d b)$ & $8.47 \pm 2.03$ & $8.86 \pm 1.57$ & $6.86 \pm 1.82$ & $9.20 \pm 3.37^{\mathrm{a}}$ & $1.87 \pm 0.72$ & $1.78 \pm 0.63$ \\
\hline$\beta(1 / \mathrm{sec})$ & $1.21 \pm 0.43$ & $1.35 \pm 0.56$ & $0.95 \pm 0.33$ & $1.38 \pm 0.82^{\mathrm{a}}$ & $0.43 \pm 0.22$ & $0.44 \pm 0.31$ \\
\hline$A \times \beta(d b / s e c)$ & $10.15 \pm 3.35$ & $11.84 \pm 2.98$ & $8.68 \pm 2.56$ & $12.14 \pm 3.59^{\mathrm{a}}$ & $1.16 \pm 0.64$ & $0.83 \pm 0.20$ \\
\hline
\end{tabular}

${ }^{\mathrm{a}} \mathrm{P}<0.05$ vs. $72 \mathrm{~h}$ after surgery. Values are expressed as the mean \pm standard deviation. MBF, myocardial blood flow. A, peak intensity of the time-perfusion intensity curve, reflecting the myocardial blood volume; $\beta$, slope of the time-perfusion intensity curve, reflecting the MBF velocity; A x $\beta$, measure of MBF.
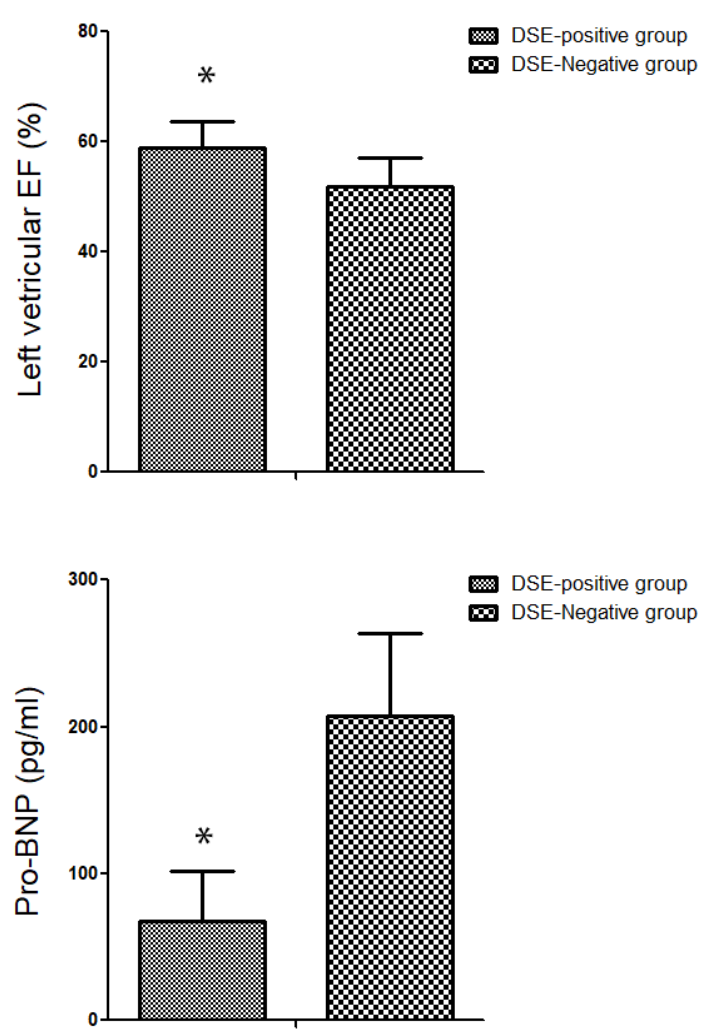

Figure 2. Left ventricular ejection fraction and pro-BNP in DSE-positive and DSE-negative group 6 months following intervention. ${ }^{*} \mathrm{P}<0.05$ vs. DSE-negative group. DSE, dobutamine stress echocardiography; pro-BNP, pro-B-type natriuretic peptide.

change was observed in the non-viable myocardium group (Table IV).

In order to evaluate the predictive value of dobutamine stress MCE for cardiac function, patients were further divided into two groups based on the change of CSI: i) Dobutamine stress echocardiography (DSE)-positive group (increase or decrease of CSI by $>0.2$ after dobutamine challenge) and ii) DSE-negative group (changes in CSI by <0.2). The results revealed that 6 months following intervention, the left ventricular ejection fraction in the DSE-positive group $(58.97 \pm 4.60 \%)$ was significantly higher compared with that in the DSE-negative group $(51.86 \pm 5.23 \%$; $\mathrm{P}<0.05$; Fig. $2 \mathrm{~A})$. With regard to the heart failure index, pro-B-type natriuretic peptide (pro-BNP), a significant decrease of pro-BNP in the DSE-positive group $(67.73 \pm 33.79 \mathrm{pg} / \mathrm{ml})$ was observed as compared with that in the DSE-negative group (207.20 $\pm 56.10 \mathrm{pg} / \mathrm{ml}$; P<0.05; Fig. 2B). Taken together, CSI may effectively predict the left ventricular function in patients with AMI.

\section{Discussion}

Either DSE or MCE has been widely applied in the evaluation of blood supply in CHD $(10,11)$. However, only few studies have been performed combining the two. Dobutamine stress testing was used to detect viable myocardium using a visual method, which was markedly affected by the subjective effect of the ultrasound physician. MCE alone may only reflect the myocardial microcirculation in the resting state and false-positives may occur. Given their respective disadvantages, the potential use of the combination of MCE with dobutamine stress testing (low-dose dobutamine stress MCE) to evaluate myocardial microcirculation perfusion and predict left ventricular function was explored in the present study. The results suggested that low-dose dobutamine stress MCE may be an effective method to evaluate myocardial microcirculation perfusion in patients with AMI after PCI. In addition, CSI as a simple semi-quantitative index is able to predict left ventricular function in patients with AMI. By combining wall motion and myocardial perfusion images, stress MCE significantly improved the diagnostic value and diagnostic accuracy for CHD. Furthermore, it is able to more accurately identify surviving and dead myocardium and detect myocardial reserve function (12-14).

Myocardial microcirculatory disorders caused by ischemia-reperfusion injury following PCI include reversible and irreversible damage. Galiuto et al (15) observed that in $50 \%$ of patients with perfusion defects who underwent MCE $24 \mathrm{~h}$ following PCI, self-healing was evident at follow-up, while the other $50 \%$ of patients had persistent perfusion defects. Repeated MCE examination prior to reperfusion therapy and early after reperfusion therapy was able to simultaneously evaluate the effects of myocardial microcirculation and interventional therapy (16).

Complete cardiomyocyte function is excitatory, conductive and contractile, and good blood perfusion and myocardial cell metabolic activity are necessary to maintain its normal 
physiological activities $(17,18)$. It is generally thought that necrotic and scarred myocardium is a type of myocardial damage, which cannot be reversed. An important characteristic of viable myocardium is that myocardial dysfunction may be reversed (19), that is, the left ventricular function index may be significantly improved following revascularization, and may benefit patients (20). It is difficult to confirm whether the perfusion defect area in the MCE examination after AMI is a complete structural injury or only a reversible injury. Therefore, a load test is required to further determine whether vasoconstriction in the perfusion defect area and abnormal dilatation of blood vessels outside the perfusion defect area exist.

In the present study, patients receiving PCI within $24 \mathrm{~h}$ of chest pain were included. It is generally thought that PCI should be performed within $24 \mathrm{~h}$ of chest pain in order to achieve timely rescue of ischemic myocardium. Furthermore, the difference in the microcirculation status after the blood supply of large vessels was restored. An increase in the values of $\mathrm{A}, \beta$ and $\mathrm{A} \times \boldsymbol{\mathrm { x }}$ was observed in normal coronary blood supply areas and a decrease in viable myocardium areas was identified after low-dose dobutamine loading.

The possible reason may be attributable to the direct expansion of the coronary artery through stimulation of the $\beta 2$ receptor by dobutamine. On the other hand, dobutamine, as a positive inotropic drug, may increase myocardial contractility, thereby increasing the myocardial oxygen demand. The increase in metabolites is able to dilate the inner diameter of the blood vessels and reduce the resistance through acting on the tiny vessels of the coronary arteries and thus increase the coronary blood flow (21). The maximum expansion of coronary vessels (coronary flow reserve) depends on the expansion ability of tiny blood vessels and the damaged coronary microvascular expansion ability is weakened, and coronary steal is likely to occur after the load. Therefore, the status of microvessels may be indirectly evaluated by detecting the coronary flow reserve by using a load test (16). In the present study, a decrease of the A, and A x $\beta$ after dobutamine challenge was revealed as compared with that in resting MCE. This is consistent with the results of Kawamoto et al (22). However, in the study by Galiuto et al (8), no significant change in the filling of contrast agent in the perfusion defect area under adenosine stress was observed. The reason may be the recovery of reversible injury of coronary microcirculation, as the load test was completed 7 days after PCI in the study by Galiuto et al (8).

Research into stress MCE is currently limited $(8,23)$. The dose and timing of the drugs and contrast agents used in the ultrasound stress test need further standardization. Dobutamine has a wide range of clinical applications. Due to its long-term use in clinical practice, its availability and side effects can be predicted, especially since low doses are considered safe. The safety of dobutamine stress MCE has been proved by several studies $(24,25)$. In the present study, no adverse effects, including abnormal heart rate, abnormal blood pressure, chest tightness, belching and dizziness during MCE, were observed.

CSI, as a simple semi-quantitative index, has been used to predict cardiac function 6 months following PCI. The present study indicated that patients with a CSI change of $>0.2$ following dobutamine loading have higher left ventricular ejection fraction and lower pro-BNP levels. For patients with microcirculation dysfunction, cardiologists may consider the addition of drugs to improve myocardial microcirculation, e.g. nicorandil, or using auxiliary Traditional Chinese Medicine.

The small sample size is one limitation of the present study. The major reason is that a high number of patients refuse PET examination, as PET is not only a radioactive detection method, but also a relatively high-cost non-medical insurance item in China. However, this suggests, from another perspective, the necessity to develop a more efficient and cost-effective method for detecting surviving myocardium. Another limitation is that the correlation between myocardial enzymes and MCE and DSE was not analyzed. In the present study, the cardiac enzyme had been tested only for the diagnosis of AMI when patients were admitted to the hospital due to chest pain. Once patients were diagnosed with AMI, PCI treatment must be performed as soon as possible. However, the timing for subsequent consultation of each individual with chest pain is not certain, so it is not guaranteed that myocardial enzyme was at its peak level. Therefore, the correlation between myocardial enzymes and MCE and DSE was not analyzed. Furthermore, only patients with single coronary artery disease were included in the present study. Further studies should involve a wide range regarding the extent or size of myocardial infarction, which may have an impact on the recovery of coronary microcirculation.

In conclusion, low-dose dobutamine stress MCE was indicated to be a safe and effective method to evaluate myocardial microcirculation perfusion in patients with acute myocardial infarction after PCI. In addition, CSI, as a simple semi-quantitative index, is able to predict left ventricular systolic function in patients with AMI.

\section{Acknowledgements}

Not applicable.

\section{Funding}

The present study was funded by the 2018 Hospital-level Project of Tianjin Chest Hospital (Tianjin, China; grant no. 2018XKC11).

\section{Availability of data and materials}

The datasets used and/or analyzed during the current study are available from the corresponding author on reasonable request.

\section{Authors' contributions}

YuL conceived the design of the study, analyzed and interpreted patient data and drafted the manuscript. XG conceived the design of the study, interpreted patient data, edited the manuscript and approved the final version of manuscript. KR was responsible for case follow-up and data analysis. YZ, YaL and YS conducted clinical examinations and participated in data analysis. All authors read and approved the final manuscript. 


\section{Ethics approval and consent to participate}

The present study was approved by the Ethics Committee of Tianjin Chest Hospital (Tianjin, China) and informed consent was obtained from all participants.

\section{Patient consent for publication}

Not applicable.

\section{Competing interests}

The authors declare that they have no competing interests.

\section{References}

1. Uyarel H, Uzunlar B, Unal Dayi S, Tartan Z, Samur H, Kasikcioglu H, Akgul O, Simsek D, Erdem I, Okmen E and Cam N: Effect of tirofiban therapy on ST segment resolution and clinical outcomes in patients with ST segment elevated acute myocardial infarction undergoing primary angioplasty. Cardiology 105: 168-175, 2006.

2. Ito H, Tomooka T, Sakai N, Yu H, Higashino Y, Fujii K, Masuyama T, Kitabatake A and Minamino T: Lack of myocardial perfusion immediately after successful thrombolysis. A predictor of poor recovery of left ventricular function in anterior myocardial infarction. Circulation 85: 1699-1705, 1992.

3. Danijela T, Jelena D, Olga P and Zorana VP: Assessment of coronary microcirculation with myocardial contrast echocardiography. Curr Pharm Des 24: 2943-2949, 2018.

4. Senior R: Role of myocardial contrast echocardiography in the clinical evaluation of acute myocardial infarction. Heart 89: 1398-1400, 2003.

5. Hayat SA and Senior R: Myocardial contrast echocardiography in ST elevation myocardial infarction: Ready for prime time? Eur Heart J 29: 299-314, 2008.

6. Aggeli C, Polytarchou K, Felekos I, Zisimos K, Venieri E, Verveniotis A, Varvarousis D, Toutouzas K, Tsiamis E and Tousoulis D: Effect of gender on the prognostic value of dobutamine stress myocardial contrast echocardiography. Hellenic J Cardiol 58: 419-424, 2017.

7. Agati L, Voci P, Autore C, Luongo R, Testa G, Mallus MT, Di Roma A, Fedele F and Dagianti A: Combined use of dobutamine echocardiography and myocardial contrast echocardiography in predicting regional dysfunction recovery after coronary revascularization in patients with recent myocardial infarction. Eur Heart J 18: 771-779, 1997.

8. Galiuto L, Locorotondo G, Paraggio L, De Caterina AR, Leone AM, Fedele E, Barchetta S, Porto I, Natale L, Rebuzzi AG, et al: Characterization of microvascular and myocardial damage within perfusion defect area at myocardial contrast echocardiography in the subacute phase of myocardial infarction. Eur Heart J Cardiovasc Imaging 13: 174-180, 2012.

9. Abdelmoneim SS, Basu A, Bernier M, Dhoble A, Abdel-Kader SS, Pellikka PA and Mulvagh SL: Detection of myocardial microvascular disease using contrast echocardiography during adenosine stress in type 2 diabetes mellitus: Prospective comparison with single-photon emission computed tomography. Diab Vasc Dis Res 8: 254-261, 2011.

10. Guiducci V, Fioroni S, Giacometti P, Manari A and Gaddi O: Early evaluation of coronary microcirculation by echocardiography with contrast medium in patients with acute myocardial infarction treated with primary percutaneous transluminal coronary angioplasty. Minerva Cardioangiol 53: 157-164, 2005.

11. Marcovitz PA and Armstrong WF: Accuracy of dobutamine stress echocardiography in detecting coronary artery disease. Am J Cardiol 69: 1269-1273, 1992.
12. Aggeli $\mathrm{C}$, Bonou $\mathrm{M}$ and Stefanadis $\mathrm{C}$ : Potential clinical applications of myocardial contrast echocardiography in evaluating myocardial perfusion in coronary artery disease. Int J Cardiol 104: 1-9, 2005.

13. Vancraeynest D, Kefer J, Hanet C, Fillee C, Beauloye C, Pasquet A, Gerber BL, Philippe M and Vanoverschelde JL: Release of cardiac bio-markers during high mechanical index contrast-enhanced echocardiography in humans. Eur Heart J 28: 1236-1241, 2007.

14. Moir S, Haluska BA, Jenkins C, Fathi R and Marwick TH: Incremental benefit of myocardial contrast to combined dipyridamole-exercise stress echocardiography for the assessment of coronary artery disease. Circulation 110: 1108-1113, 2004.

15. Galiuto L, Lombardo A, Maseri A, Santoro L, Porto I, Cianflone D, Rebuzzi AG and Crea F: Temporal evolution and functional outcome of no reflow: Sustained and spontaneously reversible patterns following successful coronary recanalisation. Heart 89: 731-737, 2003.

16. Galiuto L, Garramone B, Burzotta F, Lombardo A, Barchetta S, Rebuzzi AG and Crea F; REMEDIA Investigators: Thrombus aspiration reduces microvascular obstruction after primary coronary intervention: A myocardial contrast echocardiography substudy of the REMEDIA Trial. J Am Coll Cardiol 48: 1355-1360, 2006.

17. Arrighi JA and Dilsizian V: Multimodality imaging for assessment of myocardial viability: Nuclear, echocardiography, MR, and CT. Curr Cardiol Rep 14: 234-243, 2012.

18. Liu M, Ma Z, Guo X, Zhu J and Su J: Technetium-99m-labelled HL91 and technetium-99m-labelled MIBI SPECT imaging for the detection of ischaemic viable myocardium: A preliminary study. Clin Physiol Funct Imaging 32: 25-32, 2012.

19. Fernandes H, Sousa A, Campos J, Patrício J, Oliveira P, Vieira T, Oliveira A, Faria T, Perez B, Martins E and Pereira J: Myocardial viability assessment. Acta Med Port 24 (Suppl 4): S989-S994, 2011 (In Portuguese).

20. Kobylecka M, Maczewska J, Fronczewska-Wieniawska K, Mazurek T, Płazińska MT and Królicki L: Myocardial viability assessment in 18FDG PET/CT study (18FDG PET myocardial viability assessment). Nucl Med Rev Cent East Eur 15: 52-60, 2012.

21. Takehana K, Ruiz M, Petruzella FD, Watson DD, Beller GA and Glover DK: Response to incremental doses of dobutamine early after reperfusion is predictive of the degree of myocardial salvage in dogs with experimental acute myocardial infarction. J Am Coll Cardiol 35: 1960-1968, 2000

22. Kawamoto T, Yoshida K, Akasaka T, Hozumi T, Takagi T, Kaji S and Ueda Y: Can coronary blood flow velocity pattern after primary percutaneous transluminal coronary angioplasty [correction of angiography] predict recovery of regional left ventricular function in patients with acute myocardial infarction? Circulation 100: 339-345, 1999.

23. Taghizadeh Asl M, Mandegar MH, Roshanali F and Assadi M: Comparison of stress dobutamine echocardiography and stress dobutamine gated myocardial SPECT for the detection of viable myocardium. Nucl Med Rev Cent East Eur 17: 18-25, 2014.

24. Aggeli C, Giannopoulos G, Roussakis G, Christoforatou E, Marinos G, Toli C, Pitsavos C and Stefanadis C: Safety of myocardial flash-contrast echocardiography in combination with dobutamine stress testing for the detection of ischaemia in 5250 studies. Heart 94: 1571-1577, 2008.

25. Timperley J, Mitchell AR, Thibault H, Mirza IH and Becher H: Safety of contrast dobutamine stress echocardiography: A single center experience. J Am Soc Echocardiogr 18: $163-167,2005$

This work is licensed under a Creative Commons Attribution-NonCommercial-NoDerivatives 4.0 International (CC BY-NC-ND 4.0) License. 